\title{
MTRA-Aktionstage: Werbung für den Beruf
}

Seit dem ersten Aktionstag 2009 konnten MTRA-Schulen insgesamt rund $\mathbf{5 0 0 0}$ Besucherinnen und Besucher an knapp 40 verschiedenen Standorten willkommen heißen. Auch 2018 gestalteten engagierten Teams aus MTRA-Schülerinnen und -Schülern und ihren Lehrern abwechslungsreiche Programme.

Meist an einem Samstag öffneten 2018 deutschlandweit 20 MTRA-Schulen in Verbindung mit radiologischen, nuklearmedizinischen und strahlentherapeutischen Partnern ihre Türen und ermöglichten Einblicke in die faszinierende Welt der Radiologie. Damit soll der Beruf des/der MTRA bekannter gemacht und junge Menschen für einen modernen und spannenden technischen Beruf interessiert werden.

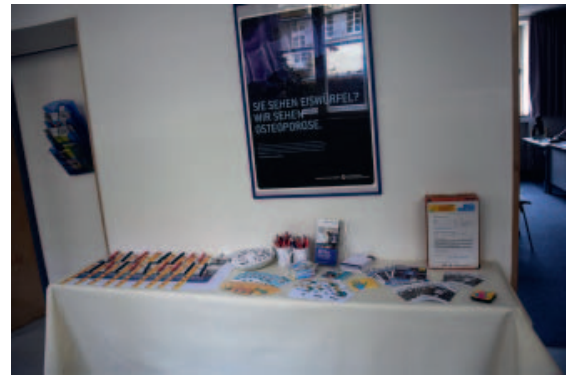

Herzlich willkommen! Die Nürnberger MTRA-Schule hielt viele Informationsmaterialien für die Interessierten bereit - und natürlich ein spannendes Programm mit Berufseinblicken. Foto: MTRA-Schule Nürnberg

\section{DIE MTRA-AKTIONSTAGE}

Tradition seit 2009: Der bundesweite MTRA-Aktionstag, organisiert von den drei Verbänden Dachverband der Technologen/-innen und Analytiker/-innen in der Medizin Deutschland e. V., Vereinigung Medizinisch-Technischer Berufe in der DRG und Deutsche Röntgengesellschaft e. V., findet jährlich im November - dem so genannten X-Ray-Monat, in dem Wilhelm Conrad Röntgen 1895 die nach ihm benannten Strahlen entdeckt hat - statt. 